\title{
PARTITION IDENTITIES ARISING FROM THETA FUNCTION IDENTITIES
}

\author{
NAYANDEEP DEKA BARUAH ${ }^{1}$ AND BRUCE C. BERNDT ${ }^{2}$
}

\begin{abstract}
In this paper, we show that certain theta function identities of Schröter and Ramanujan imply elegant partition identities.

Key Words: partitions, colored partitions, partition identities, theta-function identities, modular equations
\end{abstract}

2000 Mathematics Classification Numbers: Primary 11P83; Secondary 05A17

\section{Introduction}

H. M. Farkas and I. Kra [5] observed that certain theta function identities yield remarkably interesting identities for partition functions. Most likely, their most interesting identity for partitions is contained in the following theorem.

Theorem 1.1. Let $S$ denote the set consisting of one copy of the positive integers and one additional copy of those positive integers that are multiples of 7 . Then for each positive integer $k$, the number of partitions of $2 k$ into even elements of $S$ is equal to the number of partitions of $2 k+1$ into odd elements of $S$.

The second author of the present paper noted that Theorem 1.1 is one of five theorems of this sort that can be derived from modular equations found by both H. Schröter [7] and Ramanujan [6]. However, each of the five theorems is slightly different from the others, and we conjecture that each is unique, i.e., there are no further theorems in which, for example, "7" in Theorem 1.1, can be replaced by another prime.

The present authors found that many further modular equations and theta function identities of Ramanujan yield elegant partition identities [1]. Some of these theta function identities give new identities for $t$-cores [1]. The purpose of this paper is to continue the study begun in [4] and [1], but we emphasize that most of the theorems offered in this paper have somewhat different forms from those in the aforementioned papers. Each of our theorems arises from a theta function identity of Schröter-type. We state three of Schröter's general formulas at the end of Section 2. Each modular equation of degree $n$ is equivalent to an identity relating theta functions with argument $q$ to theta functions with argument $q^{n}$. So, we call such a theta function identity, $a$ theta function identity of degree $n$. In this paper, we do not need to make any further references to modular equations. The theta function identities that we employ can be found in Ramanujan's notebooks [6] and/or in Berndt's books [2], [3]. As in the two

\footnotetext{
${ }^{1}$ Research partially supported by BOYSCAST Fellowship grant SR/BY/M-03/05 from DST, Govt. of India.

${ }^{2}$ Research partly supported by NSA grant MSPF-03IG-124.
} 
papers cited above, we conjecture that each of the theorems that we prove is unique. Thus, for example, in Theorem 3.1 below, "3" cannot be replaced by any other prime.

\section{Notation and Preliminary Results}

Throughout this paper, we assume that $|q|<1$ and use the standard notation

$$
(a ; q)_{\infty}:=\prod_{n=0}^{\infty}\left(1-a q^{n}\right)
$$

and

$$
\left(a_{1}, a_{2}, \ldots, a_{k} ; q\right)_{\infty}:=\left(a_{1} ; q\right)_{\infty}\left(a_{2} ; q\right)_{\infty}, \cdots,\left(a_{k} ; q\right)_{\infty} .
$$

For $|a b|<1$, Ramanujan's general theta-function $f(a, b)$ is defined by

$$
f(a, b)=\sum_{n=-\infty}^{\infty} a^{n(n+1) / 2} b^{n(n-1) / 2} .
$$

Jacobi's famous triple product identity [2, p. 35, Entry 19] is given by

$$
f(a, b)=(-a ; a b)_{\infty}(-b ; a b)_{\infty}(a b ; a b)_{\infty} .
$$

The three most important special cases of $f(a, b)$ are

$$
\begin{aligned}
& \phi(q):=f(q, q)=\sum_{n=-\infty}^{\infty} q^{n^{2}}=\left(-q ; q^{2}\right)_{\infty}^{2}\left(q^{2} ; q^{2}\right)_{\infty}, \\
& \psi(q):=f\left(q, q^{3}\right)=\sum_{n=0}^{\infty} q^{n(n+1) / 2}=\frac{\left(q^{2} ; q^{2}\right)_{\infty}}{\left(q ; q^{2}\right)_{\infty}}
\end{aligned}
$$

and

$$
f(-q):=f\left(-q,-q^{2}\right)=\sum_{n=-\infty}^{\infty}(-1)^{n} q^{n(3 n-1) / 2}=(q ; q)_{\infty},
$$

where the product representation in (2.4)-(2.6) arise from (2.3). The last equality in (2.6) is Euler's famous pentagonal number theorem. In the sequel, we use many times another famous identity of Euler, namely,

$$
\left(q ; q^{2}\right)_{\infty}^{-1}=(-q ; q)_{\infty}
$$

After Ramanujan, we also define

$$
\chi(q):=\left(-q ; q^{2}\right)_{\infty} .
$$

The functions $\phi$ and $\psi$ satisfy the following relations [2, p. 39, Entry 25].

Lemma 2.1. We have

$$
\begin{aligned}
\phi(q)+\phi(-q) & =2 \phi\left(q^{4}\right), \\
\phi(q)-\phi(-q) & =4 q \psi\left(q^{8}\right), \\
\phi(q) \phi(-q) & =\phi^{2}\left(-q^{2}\right) .
\end{aligned}
$$


We also use the following theta-function identity recorded by Ramanujan in his second notebook [6], [2, p. 49]

Lemma 2.2. We have

$$
\psi(q)=f\left(q^{6}, q^{10}\right)+q f\left(q^{2}, q^{14}\right) .
$$

The main ingredients in our proofs are theta function identities, especially Schröter's identities [7], [2, Chapter 16], which have been useful over the years in many contexts, in particular, in establishing modular equations [2, Chapters 18-20]. Several special cases worked out in [2] are quoted in the sequel. Other special cases that are needed arise from the general Schröter identities that we record below.

If $\mu$ and $\nu$ are integers such that $\mu>\nu \geq 0$ and $A$ and $B$ are arbitrary, then $[2$, p. 68],

$$
\begin{aligned}
\frac{1}{2}\left\{f\left(A q^{\mu+\nu}, q^{\mu+\nu} / A\right) f\left(B q^{\mu-\nu}, q^{\mu-\nu} / B\right)-f\left(-A q^{\mu+\nu},-q^{\mu+\nu} / A\right) f\left(-B q^{\mu-\nu},-q^{\mu-\nu} / B\right)\right\} \\
=A \sum_{m=0}^{\mu-1}(A B)^{m} q^{(2 m+1)(\mu+\nu)+2 \mu m^{2}} f\left(A^{\mu-\nu} B^{\mu+\nu} q^{(2 \mu+4 m+2)\left(\mu^{2}-\nu^{2}\right)}, \frac{q^{(2 \mu-4 m-2)\left(\mu^{2}-\nu^{2}\right)}}{A^{\mu-\nu} B^{\mu+\nu}}\right) \\
\quad \times f\left(\frac{A}{B} q^{4 \mu+2 \nu+4 \nu m}, \frac{B}{A} q^{-2 \nu-4 \nu m}\right) .
\end{aligned}
$$

If $\mu$ and $\nu$ are integers such that $\mu>\nu \geq 0$, then [2, p. 68]

$$
\begin{aligned}
& \frac{1}{2}\left\{\phi\left(q^{\mu+\nu}\right) \phi\left(q^{\mu-\nu}\right)+\phi\left(-q^{\mu+\nu}\right) \phi\left(-q^{\mu-\nu}\right)\right\} \\
& \quad=\sum_{m=0}^{\mu-1} q^{2 \mu m^{2}} f\left(q^{(2 \mu+4 m)\left(\mu^{2}-\nu^{2}\right)}, q^{(2 \mu-4 m)\left(\mu^{2}-\nu^{2}\right)}\right) f\left(q^{2 \mu+4 \nu m}, q^{2 \mu-4 \nu m}\right) .
\end{aligned}
$$

If $\mu$ is even, $\nu$ is a nonnegative integer, and $\mu>\nu$, then $[2$, p. 69]

$$
\begin{aligned}
\psi\left(q^{\mu+\nu}\right) \psi\left(q^{\mu-\nu}\right)= & \phi\left(q^{\mu\left(\mu^{2}-\nu^{2}\right)}\right) \psi\left(q^{2 \mu}\right) \\
& +\sum_{m=1}^{\mu / 2-1} q^{\mu m^{2}-\nu m} f\left(q^{(\mu+2 m)\left(\mu^{2}-\nu^{2}\right)}, q^{(\mu-2 m)\left(\mu^{2}-\nu^{2}\right)}\right) f\left(q^{2 \nu m}, q^{2 \mu-2 \nu m}\right) \\
& +q^{\mu^{3} / 4-\mu \nu / 2} \psi\left(q^{2 \mu\left(\mu^{2}-\nu^{2}\right)}\right) f\left(q^{\mu \nu}, q^{2 \mu-u \nu}\right) .
\end{aligned}
$$

\section{Partition Identities Related to Theta Function Identities of Degree 3}

Theorem 3.1. Let $A(N)$ denote the number of partitions of $4 N$ into two distinct colors with one color, say orange, appearing at most once and only in odd parts and the other color, say blue, appearing at most once and only in odd multiples of 3 . Let $B(N)$ denote the number of partitions of $4 N$ into four distinct colors, with two colors, say red and green, appearing at most once and only in multiples of 4 and the remaining two colors, say pink and violet, appearing at most once and only in odd multiples of 6 . Then, for $N \geq 1$

$$
A(N)=B(N)
$$


Proof. Setting $\mu=2$ and $\nu=1$ in (2.15), we deduce that

$$
\psi(q) \psi\left(q^{3}\right)=\phi\left(q^{6}\right) \psi\left(q^{4}\right)+q \psi\left(q^{12}\right) \phi\left(q^{2}\right) .
$$

Replacing $q$ by $-q$ in (3.1), we find that

$$
\psi(-q) \psi\left(-q^{3}\right)=\phi\left(q^{6}\right) \psi\left(q^{4}\right)-q \psi\left(q^{12}\right) \phi\left(q^{2}\right) .
$$

Adding (3.1) and (3.2), we find that

$$
\psi(q) \psi\left(q^{3}\right)+\psi(-q) \psi\left(-q^{3}\right)=2 \phi\left(q^{6}\right) \psi\left(q^{4}\right) .
$$

Writing (3.3) in $q$-products with the aid of (2.4) and (2.5), and then simplifying with the help of $(2.7)$, we arrive at

$$
\left(-q ; q^{2}\right)_{\infty}\left(-q^{3} ; q^{6}\right)_{\infty}+\left(q ; q^{2}\right)_{\infty}\left(q^{3} ; q^{6}\right)_{\infty}=2\left(-q^{4} ; q^{4}\right)_{\infty}^{2}\left(-q^{6} ; q^{12}\right)_{\infty}^{2}
$$

It is readily seen that (3.4) has the partition theoretic interpretation given in Theorem 3.1 .

\section{Example: $N=3$}

Then $A(3)=7=B(3)$, and the representations that we seek are given by

$$
\begin{aligned}
11_{o}+1_{o} & =9_{o}+3_{o}=9_{o}+3_{b}=9_{b}+3_{o}=9_{b}+3_{b}=7_{o}+5_{o}=5_{o}+3_{o}+3_{b}+1_{o}, \\
12_{r} & =12_{g}=6_{p}+6_{v}=8_{r}+4_{r}=8_{r}+4_{g}=8_{g}+4_{r}=8_{g}+4_{g} .
\end{aligned}
$$

Theorem 3.2. In the definition of $A(N)$ in Theorem 3.1, replace $4 N$ by $2 N+1$. Let $C(N)$ denote the number of partitions of $2 N$ into four distinct colors, with two colors, say red and green, appearing at most once and only in odd multiples of 2 and the remaining two colors, say pink and violet, appearing at most once and only in multiples of 12 . Then, for $N \geq 1$,

$$
A(N)=C(N)
$$

Proof. Subtracting (3.2) from (3.1), we find that

$$
\psi(q) \psi\left(q^{3}\right)-\psi(-q) \psi\left(-q^{3}\right)=2 q \phi\left(q^{2}\right) \psi\left(q^{12}\right) .
$$

The identity (3.5) was also recorded by Ramanujan in his first notebook [6, p. 230], [3, p. 356, Entry 2]. Writing (3.5) in q-products with the help of (2.4) and (2.5), and then simplifying with the aid of (2.7), we deduce that

$$
\left(-q ; q^{2}\right)_{\infty}\left(-q^{3} ; q^{6}\right)_{\infty}-\left(q ; q^{2}\right)_{\infty}\left(q^{3} ; q^{6}\right)_{\infty}=2 q\left(-q^{2} ; q^{4}\right)_{\infty}^{2}\left(-q^{12} ; q^{12}\right)_{\infty}^{2}
$$

From (3.6), we readily deduce the partition identity claimed in our theorem.

\section{Example: $N=8$}

Then $A(8)=11=C(8)$, and the required representations are given by

$$
\begin{aligned}
17_{o} & =13_{o}+3_{o}+1_{o}=13_{o}+3_{b}+1_{o}=11_{o}+5_{o}+1_{o}=11_{o}+3_{o}+3_{b}=9_{o}+7_{o}+1_{o} \\
& =9_{b}+7_{o}+1_{o}=9_{o}+5_{o}+3_{o}=9_{o}+5_{o}+3_{b}=9_{b}+5_{o}+3_{o}=9_{b}+5_{o}+3_{b}, \\
14_{r}+2_{r} & =14_{r}+2_{g}=14_{g}+2_{r}=14_{g}+2_{g}=12_{p}+2_{r}+2_{g}=12_{v}+2_{r}+2_{g}=10_{r}+6_{r} \\
& =10_{r}+6_{g}=10_{g}+6_{r}=10_{g}+6_{g}=6_{r}+6_{g}=2_{r}+2_{g} .
\end{aligned}
$$


Theorem 3.3. Let $A(N)$ denote the number of partitions of $2 N+1$ into distinct odd parts that are not multiples of 3 , with each having two colors, say orange and blue. Let $B(N)$ denote the number of partitions of $2 N$ into four distinct colors, with one color, say red, appearing at most once and only in multiples of 4, two colors, say green and violet, appearing at most once and only in multiples of 6 , and the remaining color, say pink, appearing at most once and only in multiples of 12 . Then, for $N \geq 1$,

$$
A(N)=2 B(N) \text {. }
$$

Proof. Setting $\mu=2, \nu=1, A=1$, and $B=-1$ in (2.13), we can deduce that

$$
\phi(q) \phi\left(-q^{3}\right)-\phi(-q) \phi\left(q^{3}\right)=4 q \psi\left(-q^{2}\right) \psi\left(-q^{6}\right) .
$$

Dividing both sides of $(3.7)$ by $\phi\left(q^{3}\right) \phi\left(-q^{3}\right)$ and using (2.11), we find that

$$
\frac{\phi(q)}{\phi\left(q^{3}\right)}-\frac{\phi(-q)}{\phi\left(-q^{3}\right)}=4 q \frac{\psi\left(-q^{2}\right) \psi\left(-q^{6}\right)}{\phi^{2}\left(-q^{6}\right)} .
$$

Expressing (3.8) in $q$-products with the help of (2.4) and (2.5), and then employing (2.7), we obtain

$$
\left(-q ; q^{6}\right)_{\infty}^{2}\left(-q^{5} ; q^{6}\right)_{\infty}^{2}-\left(q ; q^{6}\right)_{\infty}^{2}\left(q^{5} ; q^{6}\right)_{\infty}^{2}=4 q\left(-q^{4} ; q^{4}\right)_{\infty}\left(-q^{6} ; q^{6}\right)_{\infty}^{2}\left(-q^{12} ; q^{12}\right)_{\infty}
$$

From (3.9), we readily deduce the partition identity stated in our theorem.

Example: $N=6$

Then $A(6)=12$ and $B(6)=6$, with the relevant representations being given by

$$
\begin{aligned}
13_{o} & =13_{b}=11_{o}+1_{o}+1_{b}=11_{b}+1_{o}+1_{b}=7_{o}+5_{o}+1_{o}=7_{o}+5_{o}+1_{b}=7_{o}+5_{b}+1_{o} \\
& =7_{o}+5_{b}+1_{b}=7_{b}+5_{o}+1_{o}=7_{b}+5_{o}+1_{b}=7_{b}+5_{b}+1_{o}=7_{b}+5_{b}+1_{b}, \\
12_{r} & =12_{g}=12_{v}=12_{p}=6_{g}+6_{v}=8_{r}+4_{r} .
\end{aligned}
$$

Theorem 3.4. Let $A(N)$ denote the number of partitions of $2 N+1$ into odd parts that are not multiples of 3 , with each having two colors, say orange and blue. Let $B(N)$ denote the number of partitions of $2 N$ into four distinct colors, with two colors, say red and green, appearing at most once and only in multiples of 2, one color, say pink, appearing at most once and only in multiples of 4, and the remaining color, say violet, appearing at most once and only in multiples of 12 . Then, for $N \geq 2$,

$$
A(N)=2 B(N) \text {. }
$$

Proof. Dividing both sides of $(3.7)$ by $\phi(q) \phi(-q)$ and employing (2.11), we deduce that

$$
\frac{\phi\left(-q^{3}\right)}{\phi(-q)}-\frac{\phi\left(q^{3}\right)}{\phi(q)}=4 q \frac{\psi\left(-q^{2}\right) \psi\left(-q^{6}\right)}{\phi^{2}\left(-q^{2}\right)} .
$$

Writing (3.10) in product notation with the aid of (2.4) and (2.5), and then using (2.7), we deduce that

$$
\frac{1}{\left(q ; q^{6}\right)_{\infty}^{2}\left(q^{5} ; q^{6}\right)_{\infty}^{2}}-\frac{1}{\left(-q ; q^{6}\right)_{\infty}^{2}\left(-q^{5} ; q^{6}\right)_{\infty}^{2}}=4 q\left(-q^{2} ; q^{2}\right)_{\infty}^{2}\left(-q^{4} ; q^{4}\right)_{\infty}\left(-q^{12} ; q^{12}\right)_{\infty}
$$

From (3.11), we deduce the partition identity claimed in the theorem. 


\section{Example: $N=3$}

Then $A(3)=16, B(3)=8$, and the required representations are given by

$$
\begin{aligned}
7_{o} & =7_{b}=5_{o}+1_{o}+1_{o}=5_{o}+1_{o}+1_{b}=5_{o}+1_{b}+1_{b}=5_{b}+1_{o}+1_{o}=5_{b}+1_{o}+1_{b} \\
& =5_{b}+1_{b}+1_{b}=1_{o}+1_{o}+1_{o}+1_{o}+1_{o}+1_{o}+1_{o}=1_{o}+1_{o}+1_{o}+1_{o}+1_{o}+1_{o}+1_{b} \\
& =1_{o}+1_{o}+1_{o}+1_{o}+1_{o}+1_{b}+1_{b}=1_{o}+1_{o}+1_{o}+1_{o}+1_{b}+1_{b}+1_{b} \\
& =1_{o}+1_{o}+1_{o}+1_{b}+1_{b}+1_{b}+1_{b}=1_{o}+1_{o}+1_{b}+1_{b}+1_{b}+1_{b}+1_{b} \\
& =1_{o}+1_{b}+1_{b}+1_{b}+1_{b}+1_{b}+1_{b}=1_{b}+1_{b}+1_{b}+1_{b}+1_{b}+1_{b}+1_{b}, \\
6_{r} & =6_{g}=4_{r}+2_{r}=4_{r}+2_{g}=4_{g}+2_{g}=4_{p}+2_{r}=4_{p}+2_{g}=4_{g}+2_{r} .
\end{aligned}
$$

\section{Partition Identities Related to Theta Function Identities of Degree 5}

Theorem 4.1. Let $A(N)$ denote the number of partitions of $2 N+1$ into distinct odd parts that are not multiples of 5, with each having two colors, say orange and blue. Let $B(N)$ denote the number of partitions of $2 N$ into four distinct colors, with one color, say red, appearing at most once and only in multiples of 2 , and the remaining three colors, say green, pink, and violet, appearing at most once and only in multiples of 10. Then, for $N \geq 1$,

$$
A(N)=2 B(N)
$$

Proof. Setting $\mu=3, \nu=2, A=1$, and $B=-1$ in (2.13), we can deduce that

$$
\phi(q) \phi\left(-q^{5}\right)-\phi(-q) \phi\left(q^{5}\right)=4 q f\left(-q^{4}\right) f\left(-q^{20}\right) .
$$

Dividing both sides of (4.1) by $\phi\left(q^{5}\right) \phi\left(-q^{5}\right)$ and employing (2.11), we find that

$$
\frac{\phi(q)}{\phi\left(q^{5}\right)}-\frac{\phi(-q)}{\phi\left(-q^{5}\right)}=4 q \frac{f\left(-q^{4}\right) f\left(-q^{20}\right)}{\phi^{2}\left(-q^{10}\right)} .
$$

Expressing (4.2) in product notation with the help of (2.4) and (2.6), and then employing (2.7), we find that

$$
\begin{array}{r}
\left(-q ; q^{10}\right)_{\infty}^{2}\left(-q^{3} ; q^{10}\right)_{\infty}^{2}\left(-q^{7} ; q^{10}\right)_{\infty}^{2}\left(-q^{9} ; q^{10}\right)_{\infty}^{2}-\left(q ; q^{10}\right)_{\infty}^{2}\left(q^{3} ; q^{10}\right)_{\infty}^{2}\left(q^{7} ; q^{10}\right)_{\infty}^{2}\left(q^{9} ; q^{10}\right)_{\infty}^{2} \\
=4 q\left(-q^{2} ; q^{2}\right)_{\infty}\left(-q^{10} ; q^{10}\right)_{\infty}^{3} .
\end{array}
$$

It is now easily seen that (4.3) has the partition theoretic interpretation stated in our theorem.

\section{Example: $N=5$}

Then $A(5)=12, B(5)=6$, and the representations we seek are

$$
\begin{aligned}
11_{o} & =11_{b}=9_{o}+1_{o}+1_{b}=9_{b}+1_{o}+1_{b}=7_{o}+3_{o}+1_{o}=7_{o}+3_{o}+1_{b}=7_{o}+3_{b}+1_{o} \\
& =7_{o}+3_{b}+1_{b}=7_{b}+3_{o}+1_{o}=7_{b}+3_{o}+1_{b}=7_{b}+3_{b}+1_{o}=7_{b}+3_{b}+1_{b}, \\
10_{r} & =10_{g}=10_{p}=10_{v}=8_{r}+2_{r}=6_{r}+4_{r} .
\end{aligned}
$$

Theorem 4.2. Let $A(N)$ denote the number of partitions of $2 N+1$ into odd parts that are not multiples of 5 and each having two colors, say orange and blue. Let $B(N)$ denote the number of partitions of $2 N$ into four distinct colors, with three colors, say red, green, 
and pink, appearing at most once and only in multiples of 2 , and the remaining color, say violet, appearing at most once and only in multiples of 10 . Then, for $N \geq 1$,

$$
A(N)=2 B(N) \text {. }
$$

Proof. Dividing both sides of (4.1) by $\phi(q) \phi(-q)$ and using (2.11), we obtain the identity

$$
\frac{\phi\left(-q^{5}\right)}{\phi(-q)}-\frac{\phi\left(q^{5}\right)}{\phi(q)}=4 q \frac{f\left(-q^{4}\right) f\left(-q^{20}\right)}{\phi^{2}\left(-q^{2}\right)} .
$$

Writing (4.4) in $q$-products with the help of (2.4) and (2.6), and then employing (2.7), we deduce that

$$
\frac{1}{\left(q, q^{3}, q^{7}, q^{9} ; q^{10}\right)_{\infty}^{2}}-\frac{1}{\left(-q,-q^{3},-q^{7},-q^{9} ; q^{10}\right)_{\infty}^{2}}=4 q\left(-q^{2} ; q^{2}\right)_{\infty}^{3}\left(-q^{10} ; q^{10}\right)_{\infty}
$$

From (4.5), we deduce the partition identity claimed in the theorem.

$$
\text { Example: } N=2
$$

Then $A(2)=12, B(2)=6$, and the required partitions are

$$
\begin{aligned}
3_{o}+1_{o}+1_{o} & =3_{o}+1_{o}+1_{b}=3_{o}+1_{b}+1_{b}=3_{b}+1_{o}+1_{o}=3_{b}+1_{o}+1_{b} \\
& =3_{b}+1_{b}+1_{b}=1_{o}+1_{o}+1_{o}+1_{o}+1_{o}=1_{o}+1_{o}+1_{o}+1_{o}+1_{b} \\
& =1_{o}+1_{o}+1_{o}+1_{b}+1_{b}=1_{o}+1_{o}+1_{b}+1_{b}+1_{b} \\
& =1_{o}+1_{b}+1_{b}+1_{b}+1_{b}=1_{b}+1_{b}+1_{b}+1_{b}+1_{b}, \\
4_{r} & =4_{g}=4_{p}=2_{r}+2_{g}=2_{r}+2_{p}=2_{g}+2_{p} .
\end{aligned}
$$

\section{The Theorem of Farkas and Kra and a Theta Function Identity of Degree 7}

Our aim in this section is to demonstrate that Schröter's identity (2.13) easily leads to the theta function identity yielding Theorem 1.1 of Farkas and Kra [5].

Setting $\mu=4$ and $\nu=3$ in (2.15), we can deduce that

$$
\psi(q) \psi\left(q^{7}\right)=\phi\left(q^{28}\right) \psi\left(q^{8}\right)+q \psi\left(q^{14}\right) \psi\left(q^{2}\right)+q^{6} \psi\left(q^{56}\right) \phi\left(q^{4}\right) .
$$

Replacing $q$ by $-q$ in (5.1), and then subtracting the resulting identity from (5.1), we find that

$$
\psi(q) \psi\left(q^{7}\right)-\psi(-q) \psi\left(-q^{7}\right)=2 q \psi\left(q^{14}\right) \psi\left(q^{2}\right)
$$

Writing (5.2) in terms of $q$-products with the help of (2.5), we see that

$$
\frac{\left(q^{2} ; q^{2}\right)_{\infty}\left(q^{14} ; q^{14}\right)_{\infty}}{\left(q ; q^{2}\right)_{\infty}\left(q^{7} ; q^{14}\right)_{\infty}}-\frac{\left(q^{2} ; q^{2}\right)_{\infty}\left(q^{14} ; q^{14}\right)_{\infty}}{\left(-q ; q^{2}\right)_{\infty}\left(-q^{7} ; q^{14}\right)_{\infty}}=2 q \frac{\left(q^{4} ; q^{4}\right)_{\infty}\left(q^{28} ; q^{28}\right)_{\infty}}{\left(q^{2} ; q^{4}\right)_{\infty}\left(q^{14} ; q^{28}\right)_{\infty}}
$$

We can rewrite (5.3) with the help of (2.7) as

$$
\begin{aligned}
\left(-q ; q^{2}\right)_{\infty}\left(-q^{7} ; q^{14}\right)_{\infty}-\left(q ; q^{2}\right)_{\infty}\left(q^{7} ; q^{14}\right)_{\infty} & =2 q \frac{\left(q^{4} ; q^{4}\right)_{\infty}\left(q^{28} ; q^{28}\right)_{\infty}}{\left(q^{2} ; q^{2}\right)_{\infty}\left(q^{14} ; q^{14}\right)_{\infty}} \\
& =2 q\left(-q^{2} ; q^{2}\right)_{\infty}\left(-q^{14} ; q^{28}\right)_{\infty}
\end{aligned}
$$

It can readily be seen that (5.4) is equivalent to Theorem 1.1. 
In fact, each of the partition identities proved in [5] and [4] arises from a theta function identity that can be proved by using one of Schröter's formulas.

\section{Partition Identities Related to Theta Function Identities of Degree 15}

Theorem 6.1. Let $A(N)$ denote the number of partitions of $2 N$ into two distinct colors with one color, say orange, appearing at most once and only in odd parts and the other color, say blue, also appearing at most once and only in odd multiples of 15 . Let $B(N)$ denote the number of partitions of $2 N$ into parts congruent to $\pm 4, \pm 6, \pm 8, \pm 10, \pm 16$, $\pm 18, \pm 28$, or 30 modulo 60 with parts congruent to 30 modulo 60 having two colors. Then, for $N \geq 2$,

$$
A(N)=B(N)
$$

Proof. From Entry 9(iv) of Chapter 20 in Ramanujan's second notebook [6], [2, p. 377],

$$
\psi(q) \psi\left(q^{15}\right)+\psi(-q) \psi\left(-q^{15}\right)=2 \psi\left(q^{6}\right) \psi\left(q^{10}\right) .
$$

Writing (6.1) in $q$-products with the help of (2.5), we obtain the equivalent identity

$$
\frac{\left(q^{2} ; q^{2}\right)_{\infty}\left(q^{30} ; q^{30}\right)_{\infty}}{\left(q ; q^{2}\right)_{\infty}\left(q^{15} ; q^{30}\right)_{\infty}}+\frac{\left(q^{2} ; q^{2}\right)_{\infty}\left(q^{30} ; q^{30}\right)_{\infty}}{\left(-q ; q^{2}\right)_{\infty}\left(-q^{15} ; q^{30}\right)_{\infty}}=2 \frac{\left(q^{12} ; q^{12}\right)_{\infty}\left(q^{20} ; q^{20}\right)_{\infty}}{\left(q^{6} ; q^{12}\right)_{\infty}\left(q^{10} ; q^{20}\right)_{\infty}}
$$

We rewrite $(6.2)$ as

$$
\begin{gathered}
\left(-q ; q^{2}\right)_{\infty}\left(-q^{15} ; q^{30}\right)_{\infty}+\left(q ; q^{2}\right)_{\infty}\left(q^{15} ; q^{30}\right)_{\infty}=2 \frac{\left(q^{12} ; q^{12}\right)_{\infty}\left(q^{20} ; q^{20}\right)_{\infty}}{\left(q^{4} ; q^{4}\right)_{\infty}\left(q^{6} ; q^{12}\right)_{\infty}\left(q^{10} ; q^{20}\right)_{\infty}\left(q^{60} ; q^{60}\right)_{\infty}} \\
=\frac{2}{\left(q^{4}, q^{6}, q^{8}, q^{10}, q^{16}, q^{18}, q^{28}, q^{30}, q^{30}, q^{32}, q^{42}, q^{44}, q^{50}, q^{52}, q^{54}, q^{56} ; q^{60}\right)_{\infty}} \cdot(6.3)
\end{gathered}
$$

It can readily be seen that (6.3) has the partition theoretic interpretation claimed in our theorem.

\section{Example: $N=8$}

Then $A(8)=6=B(8)$, and the representations we want are given by

$$
\begin{aligned}
15_{o}+1_{o} & =15_{b}+1_{o}=13_{o}+3_{o}=11_{o}+5_{o}=9_{o}+7_{o}=7_{o}+5_{o}+3_{o}+1_{o}, \\
16 & =10+6=8+8=8+4+4=6+6+4=4+4+4+4 .
\end{aligned}
$$

Theorem 6.2. Let $A(N)$ denote the number of partitions of $2 N+3$ into parts congruent to $\pm 4, \pm 8, \pm 16, \pm 28$ modulo 60 or into two distinct colors with one color, say orange, appearing at most once and only in odd multiples of 3 and the other color, say blue, also appearing at most once and only in odd multiples of 5 . Let $B(N)$ denote the number of partitions of $2 N$ into two distinct colors with one color, say red, appearing at most once and only in even parts and the other color, say green, appearing at most once and only in multiples of 30 . Then, for $N \geq 1$,

$$
A(N)=B(N) \text {. }
$$


Proof. From Entry 9(i) in Chapter 20 of Ramanujan's second notebook [6], [2, p. 377],

$$
\psi\left(q^{3}\right) \psi\left(q^{5}\right)-\psi\left(-q^{3}\right) \psi\left(-q^{5}\right)=2 q^{3} \psi\left(q^{2}\right) \psi\left(q^{30}\right) .
$$

Expressing (6.4) in $q$-products with the aid of (2.5), we deduce that

$$
\frac{\left(q^{6} ; q^{6}\right)_{\infty}\left(q^{10} ; q^{10}\right)_{\infty}}{\left(q^{3} ; q^{6}\right)_{\infty}\left(q^{5} ; q^{10}\right)_{\infty}}-\frac{\left(q^{6} ; q^{6}\right)_{\infty}\left(q^{10} ; q^{10}\right)_{\infty}}{\left(-q^{3} ; q^{6}\right)_{\infty}\left(-q^{5} ; q^{10}\right)_{\infty}}=2 q^{3} \frac{\left(q^{4} ; q^{4}\right)_{\infty}\left(q^{60} ; q^{60}\right)_{\infty}}{\left(q^{2} ; q^{4}\right)_{\infty}\left(q^{30} ; q^{60}\right)_{\infty}} .
$$

Employing (2.7), we can rewrite (6.5) as

$$
\begin{aligned}
& 2 q^{3}\left(-q^{2} ; q^{2}\right)_{\infty}\left(-q^{30} ; q^{30}\right)_{\infty} \\
& =\left\{\left(-q^{3} ; q^{6}\right)_{\infty}\left(-q^{5} ; q^{10}\right)_{\infty}-\left(q^{3} ; q^{6}\right)_{\infty}\left(q^{5} ; q^{10}\right)_{\infty}\right\} \frac{\left(q^{12} ; q^{12}\right)_{\infty}\left(q^{20} ; q^{20}\right)_{\infty}}{\left(q^{4} ; q^{4}\right)_{\infty}\left(q^{60} ; q^{60}\right)_{\infty}} \\
& =\frac{\left(-q^{3} ; q^{6}\right)_{\infty}\left(-q^{5} ; q^{10}\right)_{\infty}-\left(q^{3} ; q^{6}\right)_{\infty}\left(q^{5} ; q^{10}\right)_{\infty}}{\left(q^{4}, q^{8}, q^{16}, q^{28}, q^{32}, q^{44}, q^{52}, q^{56} ; q^{60}\right)_{\infty}},
\end{aligned}
$$

which has the partition theoretic interpretation claimed in Theorem 6.2.

\section{Example: $N=9$}

Then $A(9)=8=B(9)$, with the relevant partitions being given by

$$
\begin{aligned}
21_{o} & =16+5_{o}=12+9_{o}=9_{o}+8+4=9_{o}+4+4+4 \\
& =8+8+5_{b}=8+5_{b}+4+4=5_{b}+4+4+4+4, \\
18_{r} & =16_{r}+2_{r}=14_{r}+4_{r}=12_{r}+6_{r}=12_{r}+4_{r}+2_{r} \\
& =10_{r}+8_{r}=10_{r}+6_{r}+2_{r}=8_{r}+6_{r}+4_{r} .
\end{aligned}
$$

Theorem 6.3. Let $A(N)$ denote the number of partitions of $2 N+1$ into parts congruent to $\pm 6, \pm 10, \pm 18,30$ modulo 60 , with parts congruent to 30 modulo 60 having two colors, say red and green, or into two distinct colors with one color, say orange, appearing at most once and only in odd parts and the other color, say blue, also appearing at most once and only in odd multiples of 15 . Let $B(N)$ denote the number of partitions of $2 N+3$ into parts congruent to $\pm 4, \pm 8, \pm 16, \pm 28$ modulo 60 , or into four distinct colors with two colors, say Orange and Blue, each appearing at most once and only in odd multiples of 3 , and the remaining two colors, say Red and Green, appearing at most once and only in odd multiples of 5 . Then, for $N \geq 0$,

$$
2 A(N)=B(N) \text {. }
$$

Proof. Setting $\mu=4, \nu=1$, and $A=B=1$ in (2.13), we deduce that

$$
\phi\left(q^{3}\right) \phi\left(q^{5}\right)-\phi\left(-q^{3}\right) \phi\left(-q^{5}\right)=4 q^{3} f\left(q^{2}, q^{14}\right) f\left(Q^{6}, Q^{10}\right)+4 q^{17} f\left(q^{6}, q^{10}\right) f\left(Q^{2}, Q^{14}\right),
$$

where $Q=q^{15}$. Now, from (2.12), we deduce that

$$
2 f\left(q^{6}, q^{10}\right)=\psi(q)+\psi(-q)
$$

and

$$
2 q f\left(q^{2}, q^{14}\right)=\psi(q)-\psi(-q)
$$

Employing (6.8) and (6.9) in (6.7), we find that

$$
\phi\left(q^{3}\right) \phi\left(q^{5}\right)-\phi\left(-q^{3}\right) \phi\left(-q^{5}\right)=2 q^{2}\{\psi(q) \psi(Q)-\psi(-q) \psi(-Q)\} .
$$


Writing (6.10) in product notation with the help of (2.4) and (2.5), we find that

$$
\begin{aligned}
& \left(q^{6} ; q^{6}\right)_{\infty}\left(q^{10} ; q^{10}\right)_{\infty}\left\{\left(-q^{3} ; q^{6}\right)_{\infty}^{2}\left(-q^{5} ; q^{10}\right)_{\infty}^{2}-\left(q^{3} ; q^{6}\right)_{\infty}^{2}\left(q^{5} ; q^{10}\right)_{\infty}^{2}\right\} \\
& \quad=2 q^{2}\left(q^{4} ; q^{4}\right)_{\infty}\left(q^{60} ; q^{60}\right)_{\infty}\left\{\left(-q ; q^{2}\right)_{\infty}\left(-q^{15} ; q^{30}\right)_{\infty}-\left(q ; q^{2}\right)_{\infty}\left(q^{15} ; q^{30}\right)_{\infty}\right\}
\end{aligned}
$$

We rewrite (6.11) in the form

$$
\begin{aligned}
& 2 q^{2} \frac{\left(-q ; q^{2}\right)_{\infty}\left(-q^{15} ; q^{30}\right)_{\infty}-\left(q ; q^{2}\right)_{\infty}\left(q^{15} ; q^{30}\right)_{\infty}}{\left(q^{6}, q^{10}, q^{18}, q^{30}, q^{30}, q^{32}, q^{44}, q^{52}, q^{56} ; q^{60}\right)_{\infty}} \\
&=\frac{\left(-q^{3} ; q^{6}\right)_{\infty}^{2}\left(-q^{5} ; q^{10}\right)_{\infty}^{2}-\left(q^{3} ; q^{6}\right)_{\infty}^{2}\left(q^{5} ; q^{10}\right)_{\infty}^{2}}{\left(q^{4}, q^{8}, q^{16}, q^{28}, q^{32}, q^{44}, q^{52}, q^{56} ; q^{60}\right)_{\infty}}
\end{aligned}
$$

It is readily seen that $(6.12)$ has the partition theoretic interpretation given in Theorem 6.3 .

\section{Example: $N=5$}

Then $A(5)=2, B(5)=4$, and the relevant representations are given by

$$
\begin{aligned}
11_{o} & =7_{o}+3_{o}+1_{o}, \\
8+5_{R} & =8+5_{G}=5_{R}+4+4=5_{G}+4+4 .
\end{aligned}
$$

Theorem 6.4. Let $A(N)$ denote the number of partitions of $4 N+6$ into two distinct colors with one color, say orange, appearing at most once and only in odd parts and the other color, say blue, also appearing at most once and only in odd multiples of 15 . Let $B(N)$ denote the number of partitions of $4 N$ into four distinct colors with two colors, say red and green, each appearing at most once and only in multiples of 4, and the remaining two colors, say pink and violet, each appearing at most once and only in multiples of 60 . Then, for $N \geq 1$,

$$
A(N)=B(N) \text {. }
$$

Proof. Setting $\mu=4$ and $\nu=1$ in (2.14), we find that

$$
\phi\left(q^{3}\right) \phi\left(q^{5}\right)+\phi\left(-q^{3}\right) \phi\left(-q^{5}\right)=2 \phi\left(q^{8}\right) \phi\left(Q^{8}\right)+4 q^{8} \psi\left(q^{4}\right) \psi\left(Q^{4}\right)+8 q^{32} \psi\left(q^{16}\right) \psi\left(Q^{16}\right),
$$

where $Q=q^{15}$. Adding (6.10) and (6.13), we find that

$$
\begin{aligned}
\phi\left(q^{3}\right) \phi\left(q^{5}\right)= & \phi\left(q^{8}\right) \phi\left(Q^{8}\right)+2 q^{8} \psi\left(q^{4}\right) \psi\left(Q^{4}\right)+4 q^{32} \psi\left(q^{16}\right) \psi\left(Q^{16}\right) \\
& +q^{2}\{\psi(q) \psi(Q)-\psi(-q) \psi(-Q)\} .
\end{aligned}
$$

Now, from Entry 9(iii) in Chapter 20 of Ramanujan's second notebook [6], [2, p. 377],

$$
\phi\left(q^{3}\right) \phi\left(q^{5}\right)=\phi\left(-q^{2}\right) \phi\left(-Q^{2}\right)+2 q^{2} \psi(q) \psi(Q) .
$$

Equating the right-hand sides of (6.14) and (6.15), we arrive at

$$
\begin{aligned}
\phi\left(-q^{2}\right) \phi\left(-Q^{2}\right)+q^{2} & \{\psi(q) \psi(Q)+\psi(-q) \psi(-Q)\} \\
& =\phi\left(q^{8}\right) \phi\left(Q^{8}\right)+2 q^{8} \psi\left(q^{4}\right) \psi\left(Q^{4}\right)+4 q^{32} \psi\left(q^{16}\right) \psi\left(Q^{16}\right) .
\end{aligned}
$$


Employing (2.9) and (2.10) in (6.16), we find that

$$
\phi\left(q^{2}\right) \phi\left(Q^{2}\right)-\phi\left(-q^{2}\right) \phi\left(-Q^{2}\right)+4 q^{8} \psi\left(q^{4}\right) \psi\left(Q^{4}\right)=2 q^{2}\{\psi(q) \psi(Q)+\psi(-q) \psi(-Q)\} \text {. }
$$

Writing (6.17) in product notation with the aid of (2.4) and (2.5), we arrive at

$$
\begin{aligned}
\left\{\left(-q^{2} ; q^{4}\right)_{\infty}^{2}(\right. & \left.\left.-Q^{2} ; Q^{4}\right)_{\infty}^{2}-\left(q^{2} ; q^{4}\right)_{\infty}^{2}\left(Q^{2} ; Q^{4}\right)_{\infty}^{2}\right\}\left(q^{4} ; q^{4}\right)_{\infty}\left(Q^{4} ; Q^{4}\right)_{\infty} \\
& +4 q^{8} \frac{\left(q^{8} ; q^{8}\right)_{\infty}\left(Q^{8} ; Q^{8}\right)_{\infty}}{\left(q^{4} ; q^{8}\right)_{\infty}\left(Q^{4} ; Q^{8}\right)_{\infty}} \\
= & 2 q^{2}\left\{\frac{\left(q^{2} ; q^{2}\right)_{\infty}\left(Q^{2} ; Q^{2}\right)_{\infty}}{\left(q ; q^{2}\right)_{\infty}\left(Q ; Q^{2}\right)_{\infty}}+\frac{\left(q^{2} ; q^{2}\right)_{\infty}\left(Q^{2} ; Q^{2}\right)_{\infty}}{\left(-q ; q^{2}\right)_{\infty}\left(-Q ; Q^{2}\right)_{\infty}}\right\} .
\end{aligned}
$$

Using (2.7), we can rewrite (6.18) as

$$
\begin{aligned}
\left(-q^{2} ; q^{4}\right)_{\infty}^{2}\left(-Q^{2} ; Q^{4}\right)_{\infty}^{2}- & \left(q^{2} ; q^{4}\right)_{\infty}^{2}\left(Q^{2} ; Q^{4}\right)_{\infty}^{2}+4 q^{8}\left(-q^{4} ; q^{4}\right)_{\infty}^{2}\left(-Q^{4} ; Q^{4}\right)_{\infty}^{2} \\
& =2 q^{2}\left\{\left(-q ; q^{2}\right)_{\infty}\left(-Q ; Q^{2}\right)_{\infty}+\left(q ; q^{2}\right)_{\infty}\left(Q ; Q^{2}\right)_{\infty}\right\}
\end{aligned}
$$

Now, let $a(n)$ be defined by

$$
\left(q^{2} ; q^{4}\right)_{\infty}^{2}\left(Q^{2} ; Q^{4}\right)_{\infty}^{2}=: \sum_{n=0}^{\infty} a(n) q^{2 n} .
$$

Using (6.20), we can rewrite (6.19) as

$$
\begin{aligned}
\sum_{n=0}^{\infty}(-1)^{n} a(n) q^{2 n}- & \sum_{n=0}^{\infty} a(n) q^{2 n}+4 q^{8}\left(-q^{4} ; q^{4}\right)_{\infty}^{2}\left(-Q^{4} ; Q^{4}\right)_{\infty}^{2} \\
& =2 q^{2}\left\{\left(-q ; q^{2}\right)_{\infty}\left(-Q ; Q^{2}\right)_{\infty}+\left(q ; q^{2}\right)_{\infty}\left(Q ; Q^{2}\right)_{\infty}\right\}
\end{aligned}
$$

Equating coefficients of $q^{4 N+8}$ on both sides of (6.21), we readily arrive at the partition identity claimed in our theorem.

\section{Example: $N=5$}

Then $A(5)=14=B(5)$, and the relevant representations for us are

$$
\begin{aligned}
25_{o}+1_{o} & =23_{o}+3_{o}=21_{o}+5_{o}=19_{o}+7_{o}=17_{o}+9_{o}=17_{o}+5_{o}+3_{o}+1_{o} \\
& =15_{o}+11_{o}=15_{b}+11_{o}=15_{o}+7_{o}+3_{o}+1_{o}=15_{b}+7_{o}+3_{o}+1_{o} \\
& =13_{o}+9_{o}+3_{o}+1_{o}=13_{o}+7_{o}+5_{o}+1_{o}=11_{o}+9_{o}+5_{o}+1_{o} \\
& =11_{o}+7_{o}+5_{o}+3_{o}, \\
20_{r} & =20_{g}=16_{r}+4_{r}=16_{r}+4_{g}=16_{g}+4_{g}=16_{g}+4_{g}=12_{r}+8_{r}=12_{r}+8_{g} \\
& =12_{g}+8_{r}=12_{g}+8_{g}=12_{r}+4_{r}+4_{g}=12_{g}+4_{r}+4_{g}=8_{r}+8_{g}+4_{r} \\
& =8_{r}+8_{g}+4_{g} .
\end{aligned}
$$

Theorem 6.5. Let $A(N)$ denote the number of partitions of $12 N+3$ into two distinct colors with one color, say orange, appearing at most once and only in odd multiples of 3 and the other color, say blue, also appearing at most once and only in odd multiples of 5. Let $B(N)$ denote the number of partitions of $12 N$ into four distinct colors with two colors, say red and green, each appearing at most once and only in multiples of 12 , 
and the remaining two colors, say pink and violet, each appearing at most once and only in multiples of 20 . Then, for $N \geq 1$,

$$
A(N)=B(N)
$$

Proof. From (6.10) and (6.1), we deduce that

$$
\phi\left(q^{3}\right) \phi\left(q^{5}\right)-\phi\left(-q^{3}\right) \phi\left(-q^{5}\right)+4 q^{2} \psi\left(q^{6}\right) \psi\left(q^{10}\right)=4 q^{2} \psi(q) \psi(Q),
$$

where $Q=q^{15}$. Now, replacing $q$ by $q^{2}$ in (6.22) and then employing (6.4), we arrive at

$$
\begin{aligned}
\phi\left(q^{6}\right) \phi\left(q^{10}\right) & -\phi\left(-q^{6}\right) \phi\left(-q^{10}\right)+4 q^{4} \psi\left(q^{12}\right) \psi\left(q^{20}\right) \\
& =2 q\left\{\psi\left(q^{3}\right) \psi\left(q^{5}\right)-\psi\left(-q^{3}\right) \psi\left(-q^{5}\right)\right\}
\end{aligned}
$$

Expressing (6.23) in $q$-products with the help of (2.4) and (2.5), we find that

$$
\begin{aligned}
\left\{\left(-q^{6} ; q^{12}\right)_{\infty}^{2}(\right. & \left.\left.-q^{10} ; q^{20}\right)_{\infty}^{2}-\left(q^{6} ; q^{12}\right)_{\infty}^{2}\left(q^{10} ; q^{20}\right)_{\infty}^{2}\right\}\left(q^{12} ; q^{12}\right)_{\infty}\left(q^{20} ; q^{20}\right)_{\infty} \\
& +4 q^{4} \frac{\left(q^{24} ; q^{24}\right)_{\infty}\left(q^{40} ; q^{40}\right)_{\infty}}{\left(q^{12} ; q^{24}\right)_{\infty}\left(q^{20} ; q^{40}\right)_{\infty}} \\
= & 2 q\left\{\frac{\left(q^{6} ; q^{6}\right)_{\infty}\left(q^{10} ; q^{10}\right)_{\infty}}{\left(q^{3} ; q^{6}\right)_{\infty}\left(q^{5} ; q^{10}\right)_{\infty}}-\frac{\left(q^{6} ; q^{6}\right)_{\infty}\left(q^{10} ; q^{10}\right)_{\infty}}{\left(-q^{3} ; q^{6}\right)_{\infty}\left(-q^{5} ; q^{10}\right)_{\infty}}\right\}
\end{aligned}
$$

Using (2.7), we rewrite $(6.24)$ as

$$
\begin{array}{r}
\left(-q^{6} ; q^{12}\right)_{\infty}^{2}\left(-q^{10} ; q^{20}\right)_{\infty}^{2}-\left(q^{6} ; q^{12}\right)_{\infty}^{2}\left(q^{10} ; q^{20}\right)_{\infty}^{2}+4 q^{4}\left(-q^{12} ; q^{12}\right)_{\infty}^{2}\left(-q^{20} ; q^{20}\right)_{\infty}^{2} \\
=2 q\left\{\left(-q^{3} ; q^{6}\right)_{\infty}\left(-q^{5} ; q^{10}\right)_{\infty}-\left(q^{3} ; q^{6}\right)_{\infty}\left(q^{5} ; q^{10}\right)_{\infty}\right\}
\end{array}
$$

Now, let $b(n)$ be defined by

$$
\left(q^{6} ; q^{12}\right)_{\infty}^{2}\left(q^{10} ; q^{20}\right)_{\infty}^{2}=: \sum_{n=0}^{\infty} b(n) q^{2 n}
$$

Employing (6.26), we can rewrite (6.25) as

$$
\begin{aligned}
\sum_{n=0}^{\infty}(-1)^{n} b(n) q^{2 n}- & \sum_{n=0}^{\infty} b(n) q^{2 n}+4 q^{4}\left(-q^{12} ; q^{12}\right)_{\infty}^{2}\left(-q^{20} ; q^{20}\right)_{\infty}^{2} \\
& =2 q\left\{\left(-q^{3} ; q^{6}\right)_{\infty}\left(-q^{5} ; q^{10}\right)_{\infty}-\left(q^{3} ; q^{6}\right)_{\infty}\left(q^{5} ; q^{10}\right)_{\infty}\right\}
\end{aligned}
$$

Equating coefficients of $q^{12 N+4}$ on both sides of (6.27), we arrive at the partition identity given in the theorem.

Example: $N=5$ 
Then $A(5)=20=B(5)$, and the representations we want are given by

$$
\begin{aligned}
63_{o} & =55_{b}+5_{b}+33_{o}=51_{o}+9_{o}+33_{o}=45_{o}+15_{o}+33_{o}=45_{o}+15_{b}+33_{o} \\
& =45_{b}+15_{o}+35_{o}=45_{b}+15_{b}+33_{o}=39_{o}+21_{o}+33_{o}=35_{o}+9_{o} \\
& =39_{o}+15_{b}+9_{o}=35_{b}+25_{b}+33_{o}=33_{o}+27_{o}+33_{o}=35_{o}+15_{b} \\
& =33_{o}+25_{b}+5_{b}=33_{o}+21_{o}+9_{o}=27_{o}+21_{o}+15_{o}=27_{o}+21_{o}+15_{b} \\
& =25_{b}+15_{o}+15_{b}+5_{b}+3_{o}=25_{b}+21_{o}+9_{o}+5_{b}+3_{o} \\
& =21_{o}+15_{o}+15_{b}+9_{o}+3_{o}, \\
60_{r} & =60_{g}=60_{p}=60_{v}=48_{r}+12_{r}=48_{r}+12_{g}=48_{g}+12_{r}=48_{g}+12_{g}=40_{p}+20_{p} \\
& =40_{p}+20_{v}=40_{v}+20_{p}=40_{v}+20_{v}=36_{r}+24_{r}=36_{r}+24_{g}=36_{g}+24_{r} \\
& =36_{g}+24_{g}=36_{r}+12_{r}+12_{g}=36_{g}+12_{r}+12_{g}=24_{r}+24_{g}+12_{r} \\
& =24_{r}+24_{g}+12_{g} .
\end{aligned}
$$

\section{Partition Identities Related to Theta Function Identities of Degree 27}

Theorem 7.1. Let $S$ denote the set of partitions into four distinct colors with two colors, say orange and blue, appearing at most once and the other remaining two colors, say red and green, appearing at most once and only in multiples of 27 . Let $A(N)$ denote the number of partitions of $2 N+7$ into odd elements of $S$ or into parts congruent to \pm 18 modulo 54, and let $B(N)$ denote the number of partitions of $2 N$ into even elements of $S$ or into parts congruent to \pm 18 modulo 54. Furthermore, let $C(N)$ denote the number of partitions of $2 N+6$ into even parts that are not multiples of 3 . Then, for $N \geq 1$

$$
A(N)=2(B(N)+C(N))
$$

Proof. From Entry 4(iv) in Chapter 20 in Ramanujan's second notebook [6], [2, p. 359],

$$
\phi(q) \phi\left(q^{27}\right)-\phi(-q) \phi\left(-q^{27}\right)=4 q f\left(-q^{6}\right) f\left(-q^{18}\right)+4 q^{7} \psi\left(q^{2}\right) \psi\left(q^{54}\right) .
$$

Expressing (7.1) in $q$-products with the help of (2.4)-(2.6), we find that

$$
\begin{aligned}
& \left\{\left(-q ; q^{2}\right)_{\infty}^{2}\left(-q^{27} ; q^{54}\right)_{\infty}^{2}-\left(q ; q^{2}\right)_{\infty}^{2}\left(q^{27} ; q^{54}\right)_{\infty}^{2}\right\}\left(q^{2} ; q^{2}\right)_{\infty}\left(q^{54} ; q^{54}\right)_{\infty} \\
& \quad=4 q\left(q^{6} ; q^{6}\right)_{\infty}\left(q^{18} ; q^{18}\right)_{\infty}+4 q^{7} \frac{\left(q^{4} ; q^{4}\right)_{\infty}\left(q^{108} ; q^{108}\right)_{\infty}}{\left(q^{2} ; q^{4}\right)_{\infty}\left(q^{54} ; q^{108}\right)_{\infty}}
\end{aligned}
$$

Dividing both sides of $(7.2)$ by $\left(q^{2} ; q^{2}\right)_{\infty}\left(q^{54} ; q^{54}\right)_{\infty}$ and then employing $(2.7)$, we deduce that

$$
\begin{aligned}
& \left(-q ; q^{2}\right)_{\infty}^{2}\left(-q^{27} ; q^{54}\right)_{\infty}^{2}-\left(q ; q^{2}\right)_{\infty}^{2}\left(q^{27} ; q^{54}\right)_{\infty}^{2} \\
& =4 q^{7}\left(-q^{2} ; q^{2}\right)_{\infty}^{2}\left(-q^{54} ; q^{54}\right)_{\infty}^{2}+4 q \frac{\left(q^{6} ; q^{6}\right)_{\infty}\left(q^{18} ; q^{18}\right)_{\infty}}{\left(q^{2} ; q^{2}\right)_{\infty}\left(q^{54} ; q^{54}\right)_{\infty}}
\end{aligned}
$$


We can rewrite $(7.3)$ in the form

$$
\begin{aligned}
& \frac{\left(-q ; q^{2}\right)_{\infty}^{2}\left(-q^{27} ; q^{54}\right)_{\infty}^{2}-\left(q ; q^{2}\right)_{\infty}^{2}\left(q^{27} ; q^{54}\right)_{\infty}^{2}}{\left(q^{18}, q^{36} ; q^{54}\right)_{\infty}} \\
& =4 q^{7} \frac{\left(-q^{2} ; q^{2}\right)_{\infty}^{2}\left(-q^{54} ; q^{54}\right)_{\infty}^{2}}{\left(q^{18}, q^{36} ; q^{54}\right)_{\infty}}+\frac{4 q}{\left(q^{2}, q^{4} ; q^{6}\right)_{\infty}}
\end{aligned}
$$

Equating coefficients of $q^{2 N+7}$ on both sides of (7.4), we readily deduce the partition identity claimed in the theorem.

\section{Example: $N=3$}

Then $A(3)=26, B(3)=6, C(3)=7$, and the relevant partitions are given by

$$
\begin{aligned}
13_{o} & =13_{b}=11_{o}+1_{o}+1_{b}=11_{b}+1_{o}+1_{b}=9_{o}+3_{o}+1_{o}=9_{o}+3_{o}+1_{b} \\
& =9_{o}+3_{b}+1_{o}=9_{o}+3_{b}+1_{b}=9_{b}+3_{o}+1_{o}=9_{b}+3_{o}+1_{b}=9_{b}+3_{b}+1_{o} \\
& =9_{b}+3_{b}+1_{b}=7_{o}+5_{o}+1_{o}=7_{o}+5_{o}+1_{b}=7_{o}+5_{b}+1_{o}=7_{o}+5_{b}+1_{b} \\
& =7_{b}+5_{o}+1_{o}=7_{b}+5_{o}+1_{b}=7_{b}+5_{b}+1_{o}=7_{b}+5_{b}+1_{b}=7_{o}+3_{o}+3_{b} \\
& =7_{b}+3_{o}+3_{b}=5_{o}+5_{b}+3_{o}=5_{o}+5_{b}+3_{b}=5_{o}+3_{o}+3_{b}+1_{o}+1_{b} \\
& =5_{b}+3_{o}+3_{b}+1_{o}+1_{b}, \\
6_{o} & =6_{b}=4_{o}+2_{o}=4_{o}+2_{b}=4_{b}+2_{o}=4_{b}+2_{b}, \\
10+2 & =8+4=8+2+2=4+4+4=4+4+2+2 \\
& =4+2+2+2+2=2+2+2+2+2 .
\end{aligned}
$$

\section{Partition Identities Related to Theta Function Identities of Degree 35}

Theorem 8.1. Let $S$ denote the set of partitions into four distinct colors with two colors, say orange and blue, appearing at most once, and the other remaining two colors, say red and green, appearing at most once and only in multiples of 35 . Let $A(N)$ denote the number of partitions of $2 N+1$ into odd elements of $S$, and let $B(N)$ denote the number of partitions of $2 N-8$ into even elements of $S$. Furthermore, let $C(N)$ denote the number of partitions of $2 N$ into even parts that are not multiples of 5 or 7 . Then, for $N \geq 5$,

$$
A(N)=2(B(N)+C(N)) .
$$

Proof. From Entry 17(i) in Chapter 20 of Ramanujan's second notebook [6], [2, p. 417],

$$
\phi(q) \phi\left(q^{35}\right)-\phi(-q) \phi\left(-q^{35}\right)-4 q^{9} \psi\left(q^{2}\right) \psi\left(q^{70}\right)=4 q f\left(-q^{10}\right) f\left(-q^{14}\right) .
$$

Writing (8.1) in product notation with the help of (2.4)-(2.6), we find that

$$
\begin{aligned}
& \left\{\left(-q ; q^{2}\right)_{\infty}^{2}\left(-q^{35} ; q^{70}\right)_{\infty}^{2}-\left(q ; q^{2}\right)_{\infty}^{2}\left(q^{35} ; q^{70}\right)_{\infty}^{2}\right\}\left(q^{2} ; q^{2}\right)_{\infty}\left(q^{70} ; q^{70}\right)_{\infty} \\
& -4 q^{9} \frac{\left(q^{4} ; q^{4}\right)_{\infty}\left(q^{140} ; q^{140}\right)_{\infty}}{\left(q^{2} ; q^{4}\right)_{\infty}\left(q^{70} ; q^{140}\right)_{\infty}}=4 q\left(q^{10} ; q^{10}\right)_{\infty}\left(q^{14} ; q^{14}\right)_{\infty}
\end{aligned}
$$


Dividing both sides of $(8.2)$ by $\left(q^{2} ; q^{2}\right)_{\infty}\left(q^{70} ; q^{70}\right)_{\infty}$ and then employing $(2.7)$, we deduce that

$$
\begin{aligned}
\left(-q ; q^{2}\right)_{\infty}^{2}\left(-q^{35} ; q^{70}\right)_{\infty}^{2} & -\left(q ; q^{2}\right)_{\infty}^{2}\left(q^{35} ; q^{70}\right)_{\infty}^{2}-4 q^{9}\left(-q^{2} ; q^{2}\right)_{\infty}^{2}\left(-q^{70} ; q^{70}\right)_{\infty}^{2} \\
& =4 q \frac{\left(q^{10} ; q^{10}\right)_{\infty}\left(q^{14} ; q^{14}\right)_{\infty}}{\left(q^{2} ; q^{2}\right)_{\infty}\left(q^{70} ; q^{70}\right)_{\infty}}
\end{aligned}
$$

From (8.3), we readily deduce the partition identity claimed in the theorem.

Example: $N=5$

Then $A(5)=16, B(5)=2, C(5)=6$, and the desired representations are given by

$$
\begin{aligned}
11_{o} & =11_{b}=9_{o}+1_{o}+1_{b}=9_{b}+1_{o}+1_{b}=7_{o}+3_{o}+1_{o}=7_{o}+3_{o}+1_{b}=7_{o}+3_{b}+1_{o} \\
& =7_{o}+3_{b}+1_{b}=7_{b}+3_{o}+1_{o}=7_{b}+3_{o}+1_{b}=7_{b}+3_{b}+1_{o}=7_{b}+3_{b}+1_{b} \\
& =5_{o}+5_{b}+1_{o}=5_{o}+5_{b}+1_{b}=5_{o}+3_{o}+3_{b}=5_{b}+3_{o}+3_{b}, \\
2_{o} & =2_{b}, \\
8+2 & =6+4=6+2+2=4+4+2=4+2+2+2=2+2+2+2+2 .
\end{aligned}
$$

Theorem 8.2. Let $S$ denote the set of partitions into four distinct colors with two colors, say orange and blue, each appearing at most once and only in multiples of 5, and the other remaining two colors, say red and green, appearing at most once and only in multiples of 7 . Let $A(N)$ denote the number of partitions of $2 N+3$ into either odd elements of $S$ or into even parts not congruent to multiples of 5 or 7 modulo 70 , and let $B(N)$ denote the number of partitions of $2 N$ into either even elements of $S$ or into even parts not congruent to multiples of 5 or 7 modulo 70 . Then, for $N \geq 1$,

$$
A(N)=2 B(N)
$$

Proof. The following theta-function identity was recorded by Ramanujan in Chapter 20 of his second notebook [6], [2, p. 417, Entry 17(ii)], namely,

$$
\phi\left(q^{5}\right) \phi\left(q^{7}\right)-\phi\left(-q^{5}\right) \phi\left(-q^{7}\right)=4 q^{3} \psi\left(q^{10}\right) \psi\left(q^{14}\right)-4 q^{3} f\left(-q^{2}\right) f\left(-q^{70}\right) .
$$

Writing (8.4) in $q$-products with the help of (2.4)-(2.6), we find that

$$
\begin{aligned}
\left\{\left(-q^{5} ; q^{10}\right)_{\infty}^{2}\left(-q^{7} ; q^{14}\right)_{\infty}^{2}-\right. & \left.\left(q^{5} ; q^{10}\right)_{\infty}^{2}\left(q^{7} ; q^{14}\right)_{\infty}^{2}\right\}\left(q^{10} ; q^{10}\right)_{\infty}\left(q^{14} ; q^{14}\right)_{\infty} \\
& =4 q^{3} \frac{\left(q^{20} ; q^{20}\right)_{\infty}\left(q^{28} ; q^{28}\right)_{\infty}}{\left(q^{10} ; q^{20}\right)_{\infty}\left(q^{14} ; q^{28}\right)_{\infty}}-4 q^{3}\left(q^{2} ; q^{2}\right)_{\infty}\left(q^{70} ; q^{70}\right)_{\infty}
\end{aligned}
$$

Dividing both sides of $(8.5)$ by $\left(q^{10} ; q^{10}\right)_{\infty}\left(q^{14} ; q^{14}\right)_{\infty}$ and then employing $(2.7)$, we find that

$$
\begin{aligned}
\left(-q^{5} ; q^{10}\right)_{\infty}^{2} & \left(-q^{7} ; q^{14}\right)_{\infty}^{2}-\left(q^{5} ; q^{10}\right)_{\infty}^{2}\left(q^{7} ; q^{14}\right)_{\infty}^{2} \\
& =4 q^{3}\left(-q^{10} ; q^{10}\right)_{\infty}^{2}\left(-q^{14} ; q^{14}\right)_{\infty}^{2}-4 q^{3} \frac{\left(q^{2} ; q^{2}\right)_{\infty}\left(q^{70} ; q^{70}\right)_{\infty}}{\left(q^{10} ; q^{10}\right)_{\infty}\left(q^{14} ; q^{14}\right)_{\infty}}
\end{aligned}
$$


We can rewrite $(8.6)$ as

$$
\begin{aligned}
\left\{\left(-q^{5} ; q^{10}\right)_{\infty}^{2}(\right. & \left.\left.-q^{7} ; q^{14}\right)_{\infty}^{2}-\left(q^{5} ; q^{10}\right)_{\infty}^{2}\left(q^{7} ; q^{14}\right)_{\infty}^{2}\right\} \frac{\left(q^{10} ; q^{10}\right)_{\infty}\left(q^{14} ; q^{14}\right)_{\infty}}{\left(q^{2} ; q^{2}\right)_{\infty}\left(q^{70} ; q^{70}\right)_{\infty}} \\
& =4 q^{3}\left(-q^{10} ; q^{10}\right)_{\infty}^{2}\left(-q^{14} ; q^{14}\right)_{\infty}^{2} \frac{\left(q^{10} ; q^{10}\right)_{\infty}\left(q^{14} ; q^{14}\right)_{\infty}}{\left(q^{2} ; q^{2}\right)_{\infty}\left(q^{70} ; q^{70}\right)_{\infty}}-4 q^{3}
\end{aligned}
$$

from which we readily arrive at the partition identity claimed in our theorem.

Example: $N=6$

Then $A(6)=24, B(6)=12$, and the relevant partitions are given by

$$
\begin{aligned}
15_{o} & =15_{b}=8+7_{r}=8+7_{g}=8+5_{o}+2=8+5_{b}+2=7_{r}+6+2=7_{g}+6+2 \\
& =7_{r}+4+4=7_{g}+4+4=7_{r}+4+2+2=7_{g}+4+2+2=7_{r}+2+2+2+2 \\
& =7_{g}+2+2+2+2=6+5_{o}+4=6+5_{b}+4=6+5_{o}+2+2=6+5 b+2+2 \\
& =5_{o}+4+4+2=5_{b}+4+4+2=5_{o}+4+2+2+2=5_{b}+4+2+2+2 \\
& =5_{o}+2+2+2+2+2=5_{b}+2+2+2+2+2, \\
12 & =10_{o}+2=10_{b}+2=8+4=8+2+2=6+6=6+4+2=6+2+2+2 \\
& =4+4+4=4+4+2+2=4+2+2+2+2=2+2+2+2+2+2 .
\end{aligned}
$$

\section{Partition Identities Arising from Theta Function Identities of Degrees 39,55 , and 63}

Theorem 9.1. Let $\nu=5,3$, or 1 and define $\epsilon=0$ if $\nu=5$ or 3 , and $\epsilon=1$ if $\nu=1$. Let $A(N)$ denote the number of partitions of $2 N+6+\epsilon-\left(\nu^{2}-1\right) / 8$ into parts that are multiples of 4 but not multiples of $4(8-\nu)$ or $4(8+\nu)$, or into two distinct colors with one color, say orange, appearing at most once and only in odd multiples of $8-\nu$ and the other color, say blue, appearing at most once and only in odd multiples of $8+\nu$. Let $B(N)$ denote the number of partitions of $2 N+\epsilon$ into two distinct colors with one color, say red, appearing at most once and only in odd parts and the other color, say green, appearing at most once and only in odd multiples of $64-\nu^{2}$. Then, for $N \geq 2$,

$$
A(N)=B(N) \text {. }
$$

Proof. The three parts of the corollary in Section 37 of Chapter 16 of Ramanujan's second notebook [6], [2, p. 74] may be collectively written as

$$
\psi\left(q^{8-\nu}\right) \psi\left(q^{8+\nu}\right)-\psi\left(-q^{8-\nu}\right) \psi\left(-q^{8+\nu}\right)=q^{6-\left(\nu^{2}-1\right) / 8}\{\psi(q) \psi(Q) \pm \psi(-q) \psi(-Q)\}
$$

where $\nu=5,3,1, Q=q^{64-\nu^{2}}$, and where the plus sign is chosen in the first two cases and the minus sign is chosen in the last case. Writing (9.1) in $q$-products with the help of (2.5), we find that

$$
\begin{aligned}
& \frac{\left(q^{2(8-\nu)} ; q^{2(8-\nu)}\right)_{\infty}\left(q^{2(8+\nu)} ; q^{2(8+\nu)}\right)_{\infty}}{\left(q^{8-\nu} ; q^{2(8-\nu)}\right)_{\infty}\left(q^{8+\nu} ; q^{2(8+\nu)}\right)_{\infty}}-\frac{\left(q^{2(8-\nu)} ; q^{2(8-\nu)}\right)_{\infty}\left(q^{2(8+\nu)} ; q^{2(8+\nu)}\right)_{\infty}}{\left(-q^{8-\nu} ; q^{2(8-\nu)}\right)_{\infty}\left(-q^{8+\nu} ; q^{2(8+\nu)}\right)_{\infty}} \\
& =q^{6-\left(\nu^{2}-1\right) / 8}\left\{\frac{\left(q^{2} ; q^{2}\right)_{\infty}\left(Q^{2} ; Q^{2}\right)_{\infty}}{\left(q ; q^{2}\right)_{\infty}\left(Q ; Q^{2}\right)_{\infty}} \pm \frac{\left(q^{2} ; q^{2}\right)_{\infty}\left(Q^{2} ; Q^{2}\right)_{\infty}}{\left(-q ; q^{2}\right)_{\infty}\left(-Q ; Q^{2}\right)_{\infty}}\right\} .
\end{aligned}
$$


With the help of (2.7), we rewrite (9.2) in the form

$$
\begin{aligned}
& \frac{\left(q^{4(8-\nu)} ; q^{4(8-\nu)}\right)_{\infty}\left(q^{4(8+\nu)} ; q^{4(8+\nu)}\right)_{\infty}}{\left(q^{4} ; q^{4}\right)_{\infty}\left(Q^{4} ; Q^{4}\right)_{\infty}}\left\{\left(-q^{8-\nu} ; q^{2(8-\nu)}\right)_{\infty}\left(-q^{8+\nu} ; q^{2(8+\nu)}\right)_{\infty}\right. \\
& \left.-\left(q^{8-\nu} ; q^{2(8-\nu)}\right)_{\infty}\left(q^{8+\nu} ; q^{2(8+\nu)}\right)_{\infty}\right\} \\
& =q^{6-\left(\nu^{2}-1\right) / 8}\left\{\left(-q ; q^{2}\right)_{\infty}\left(-Q ; Q^{2}\right)_{\infty} \pm\left(q ; q^{2}\right)_{\infty}\left(Q ; Q^{2}\right)_{\infty}\right\} .
\end{aligned}
$$

We note that $6-\left(\nu^{2}-1\right) / 8$ is odd when $\nu=5$ or 3 and even when $\nu=1$. Thus, taking $\epsilon=0$ in the first two cases and $\epsilon=1$ in the last case, and then equating coefficients of $2 N+6+\epsilon-\left(\nu^{2}-1\right) / 8$ on both sides of (9.3), we arrive at the partition identity claimed in the theorem.

\section{Example: $N=8, \nu=5$}

Then $A(8)=5=B(8)$, and the relevant partitions are given by

$$
\begin{aligned}
& 16+3_{o}=15_{o}+4=8+8+3_{o}=8+4+4+3_{o}=4+4+4+4+3_{o}, \\
& 15_{r}+1_{r}=13_{r}+3_{r}=11_{r}+5_{r}=9_{r}+7_{r}=7_{r}+5_{r}+3_{r}+1_{r} .
\end{aligned}
$$

Theorem 9.2. Let $S_{1}$ denote the set of partitions into four distinct colors with two colors, say orange and blue, each appearing at most once and the remaining two colors, say red and green, appearing at most once and only in multiples of $64-\nu^{2}$. Let $S_{2}$ denote the set of partitions into four distinct colors with two colors, say Orange and Blue, each appearing at most once and only in multiples of $8-\nu$ and the remaining two colors, say Red and Green, appearing at most once and only in multiples of $8+\nu$. Let $A(N)$ denote the number of partitions of $2 N+16-\left(\nu^{2}-1\right) / 4$ into odd elements of $S_{1}$, and let $B(N)$ denote the number of partitions of $2 N$ into even elements of $S_{1}$. Furthermore, let $C(N)$ denote the number of partitions of $2 N+16-\left(\nu^{2}-1\right) / 4$ either into even parts that are not multiples of $8-\nu$ or $8+\nu$, or into odd elements of $S_{2}$, and let $D(N)$ denote the number of partitions of $2 N+12-\left(\nu^{2}-1\right) / 4$ either into even parts that are not multiples of $8-\nu$ or $8+\nu$, or into even elements of $S_{2}$. Then, for $N \geq 1$,

$$
A(N)+2 B(N)=C(N)+2 D(N) .
$$

Proof. We begin by recalling the following identity found by Berndt [2, p. 429]. If $\nu=5,3$, or 1 and $Q=q^{64-\nu^{2}}$, then

$$
\begin{aligned}
\phi\left(q^{8+\nu}\right) \phi\left(q^{8-\nu}\right)+ & \phi\left(-q^{8+\nu}\right) \phi\left(-q^{8-\nu}\right)+4 q^{4} \psi\left(q^{16+2 \nu}\right) \psi\left(q^{16-2 \nu}\right) \\
& =\phi(q) \phi(Q)+\phi(-q) \phi(-Q)+4 q^{16-\left(\nu^{2}-1\right) / 4} \psi\left(q^{2}\right) \psi\left(Q^{2}\right) .
\end{aligned}
$$

Writing (9.4) in product notation with the aid of (2.4) and (2.5), we find that

$$
\begin{aligned}
\{( & \left.\left.-q^{8-\nu} ; q^{2(8-\nu)}\right)_{\infty}^{2}\left(-q^{8+\nu} ; q^{2(8+\nu)}\right)_{\infty}^{2}+\left(q^{8-\nu} ; q^{2(8-\nu)}\right)_{\infty}^{2}\left(q^{8+\nu} ; q^{2(8+\nu)}\right)_{\infty}^{2}\right\} \\
& \times\left(q^{2(8-\nu)} ; q^{2(8-\nu)}\right)_{\infty}\left(q^{2(8+\nu)} ; q^{2(8+\nu)}\right)_{\infty} \\
& +4 q^{4} \frac{\left(q^{4(8-\nu)} ; q^{4(8-\nu)}\right)_{\infty}\left(q^{4(8+\nu)} ; q^{4(8+\nu)}\right)_{\infty}}{\left(q^{2(8-\nu)} ; q^{4(8-\nu)}\right)_{\infty}\left(q^{2(8+\nu)} ; q^{4(8+\nu)}\right)_{\infty}} \\
= & \left\{\left(-q ; q^{2}\right)_{\infty}^{2}\left(-Q ; Q^{2}\right)_{\infty}^{2}+\left(q ; q^{2}\right)_{\infty}^{2}\left(Q ; Q^{2}\right)_{\infty}^{2}\right\}\left(q^{2} ; q^{2}\right)_{\infty}\left(q^{78} ; q^{78}\right)_{\infty}
\end{aligned}
$$




$$
+4 q^{16-\left(\nu^{2}-1\right) / 4} \frac{\left(q^{4} ; q^{4}\right)_{\infty}\left(Q^{4} ; Q^{4}\right)_{\infty}}{\left(q^{2} ; q^{4}\right)_{\infty}\left(Q^{2} ; Q^{4}\right)_{\infty}} .
$$

Using (2.7), we can rewrite (9.5) in the form

$$
\begin{aligned}
& \frac{\left(q^{2(8-\nu)} ; q^{2(8-\nu)}\right)_{\infty}\left(q^{2(8+\nu)} ; q^{2(8+\nu)}\right)_{\infty}}{\left(q^{2} ; q^{2}\right)_{\infty}\left(Q^{2} ; Q^{2}\right)_{\infty}}\left\{\left(-q^{8-\nu} ; q^{2(8-\nu)}\right)_{\infty}^{2}\left(-q^{8+\nu} ; q^{2(8+\nu)}\right)_{\infty}^{2}\right. \\
& \left.+\left(q^{8-\nu} ; q^{2(8-\nu)}\right)_{\infty}^{2}\left(q^{8+\nu} ; q^{2(8+\nu)}\right)_{\infty}^{2}+4 q^{4}\left(-q^{2(8-\nu)} ; q^{2(8-\nu)}\right)_{\infty}^{2}\left(-q^{2(8+\nu)} ; q^{2(8+\nu)}\right)_{\infty}^{2}\right\} \\
& =\left(-q ; q^{2}\right)_{\infty}^{2}\left(-Q ; Q^{2}\right)_{\infty}^{2}+\left(q ; q^{2}\right)_{\infty}^{2}\left(Q ; Q^{2}\right)_{\infty}^{2}+4 q^{16-\left(\nu^{2}-1\right) / 4}\left(-q^{2} ; q^{2}\right)_{\infty}^{2}\left(-Q^{2} ; Q^{2}\right)_{\infty}^{2}
\end{aligned}
$$

Equating the coefficients of $q^{2 N+16-\left(\nu^{2}-1\right) / 4}$ on both sides of (9.6), we readily arrive at the partition identity claimed in our theorem.

\section{Example: $N=2, \nu=5$}

Then $A(2)=29, B(2)=3, C(2)=17$, and $D(2)=9$, with the relevant representations being given by

$$
\begin{aligned}
& 13_{o}+1_{o}=13_{o}+1_{b}=13_{b}+1_{o}=13_{b}+1_{b}=11_{o}+3_{o}=11_{o}+3_{b}=11_{b}+3_{o}=11_{b}+3_{b} \\
& =9_{o}+5_{o}=9_{o}+5_{b}=9_{b}+5_{o}=9_{b}+5_{b}=9_{o}+3_{o}+1_{o}+1_{b}=9_{o}+3_{b}+1_{o}+1_{b} \\
& =9_{b}+3_{o}+1_{o}+1_{b}=9_{b}+3_{b}+1_{o}+1_{b}=7_{o}+7_{b}=7_{o}+5_{o}+1_{o}+1_{b} \\
& =7_{o}+5_{b}+1_{o}+1_{b}=7_{b}+5_{o}+1_{o}+1_{b}=7_{b}+5_{b}+1_{o}+1_{b}=7_{o}+3_{o}+3_{b}+1_{o} \\
& =7_{o}+3_{o}+3_{b}+1_{b}=7_{b}+3_{o}+3_{b}+1_{o}=7_{b}+3_{o}+3_{b}+1_{b}=5_{o}+5_{b}+3_{o}+1_{o} \\
& =5_{o}+5_{b}+3_{o}+1_{b}=5_{o}+5_{b}+3_{b}+1_{o}=5_{o}+5_{b}+3_{b}+1_{b}, \\
& 4_{o}=4_{b}=2_{o}+2_{b} \text {, } \\
& 14=10+4=10+2+2=9_{O}+3_{O}+2=9_{O}+3_{B}+2=9_{B}+3_{O}+2 \\
& =9_{B}+3_{B}+2=8+4+2=8+3_{O}+3_{B}=8+2+2+2 \\
& =4+4+4+2=4+4+3_{O}+3_{B}=4+4+2+2+2 \\
& =4+3_{O}+3_{B}+2+2=4+2+2+2+2+2 \\
& =3_{O}+3_{B}+2+2+2+2=2+2+2+2+2+2+2 \text {, } \\
& 10=8+2=6_{O}+4=6_{B}+4=6_{O}+2+2=6_{B}+2+2 \\
& =4+4+2=4+2+2+2=2+2+2+2+2 \text {. }
\end{aligned}
$$

\section{REFERENCES}

[1] N. D. Baruah and B. C. Berndt, Partition identities and Ramanujan's modular equations, submitted for publication.

[2] B. C. Berndt, Ramanujan's Notebooks, Part III, Springer-Verlag, New York, 1991.

[3] B. C. Berndt, Ramanujan's Notebooks, Part V, Springer-Verlag, New York, 1998.

[4] B. C. Berndt, Partition-theoretic interpretations of certain modular equations of Schröter, Russell, and Ramanujan, Ann. of Combin., to appear.

[5] H. M. Farkas and I. Kra, Partitions and theta constant identities, in The Mathematics of Leon Ehrenpreis, Contemp. Math. No. 251, American Mathematical Society, Providence, RI, 2000, 197-203.

[6] S. Ramanujan, Notebooks (2 volumes), Tata Institute of Fundamental Research, Bombay, 1957. 
[7] H. Schröter, De aequationibus modularibus, Dissertatio Inauguralis, Albertina Litterarum Universitate, Regiomonti, 1854.

Department of Mathematics, University of Illinois at Urbana-Champaign, 1409 West Green St., Urbana, IL 61801, USA

E-mail address: nbaruah@uiuc.edu

Department of Mathematics, University of Illinois at Urbana-Champaign, 1409 West Green St., Urbana, IL 61801, USA

E-mail address: berndt@math.uiuc.edu 